\title{
Sucesos vitales estresantes, ansiedad y depresión en estudiantes de una universidad privada de Bucaramanga
}

\author{
Andres Julian Usuga Jerez \\ Psicólogo \\ Universidad Pontificia Bolivariana, Colombia \\ Correo electrónico: andres.usuga.jerez@gmail.com
}

Nancy Viviana Lemos Ramírez PhD. Psicología Clínica Universidad Pontificia Bolivariana, Colombia Correo electrónico: nancy.lemos@upb.edu.co

\section{Jessica Lizeth Pinzón Ardila}

Psicóloga

Universidad Pontificia Bolivariana, Colombia Correo electrónico: jessica.pinzon.2015@upb.edu.co

\section{Paula Fernanda Pérez Rivero}

Especialista en Psicología Clínica Universidad Pontificia Bolivariana, Colombia Correo electrónico: paula.perez@upb.edu.co

\section{Ana Fernanda Uribe Rodríguez \\ PhD. Psicología Clínica y Salud Universidad Pontificia Bolivariana, Colombia}

\section{Resumen}

El objetivo de la investigación fue evaluar la relación entre los niveles de estrés, la valencia y la predictibilidad de sucesos vitales en relación con el índice de ansiedad y depresión de estudiantes de una universidad privada en el nororiente de Colombia. Se trabajó bajo una metodología cuantitativa, con diseño transversal de tipo no experimental y alcance descriptivo-correlacional. La muestra fue de carácter no probabilístico a conveniencia constituida por un $33.7 \%(n=186)$ de hombres y $66.3 \%$ (n = 366) de mujeres, con una edad promedio de 18.9 años. Las variables del estudio se evaluaron con: el Inventario de Depresión de Beck II, el Inventario de Ansiedad de Beck y el Cuestionario de Sucesos Vitales. Se encontraron correlaciones estadísticamente significativas entre los niveles de ansiedad y depresión con la valencia negativa y la predictibilidad del suceso. Estos resultados apoyan la teoría de indefensión-desesperanza y discrepan de evidencia científica existente sobre esta temática.

Estrés, universitarios, sucesos vitales, ansiedad, depresión.

4 Para citar este artículo: Usuga-Jerez, A., Lemos-Ramírez, N., Pinzón-Ardila, J., Pérez-Rivera, P., \& Uribe-Rodríguez, A.F. (2021). Sucesos vitales estresantes, ansiedad y depresión en estudiantes de una universidad privada de Bucaramanga. Informes Psicológicos, 21(2), pp. 61-74 http://dx.doi.org/10.18566/ infpsic.v21n2a04 


\title{
Stressful life events, anxiety and depression in students of a private university in Bucaramanga
}

\begin{abstract}
The objective of the research was to evaluate the relationship between stress levels, valence and predictability of life events in relation to the anxiety and depression index of students from a private university in northeastern Colombia. We worked under a quantitative methodology, with a non-experimental cross-sectional design and a descriptive-correlational scope. The sample was nonprobabilistic at convenience, consisting of $33.7 \%(n=186)$ of men and $66.3 \%(n=366)$ of women, with an average age of 18.9 years. The study variables were evaluated with the following: The Beck Depression Inventory II, the Beck Anxiety Inventory and the Vital Events Questionnaire. Statistically significant correlations were found between levels of anxiety and depression with negative valence and the predictability of the event. These results support the helplessness-hopelessness theory and disagree with the existing scientific evidence on this issue.
\end{abstract}

Keywords

Stress, university students, life events, anxiety, depression.

\section{Eventos de vida estressantes, ansiedade e depressão em alunos de uma universidade particular em Bucaramanga}

\begin{abstract}
Resumo
0 objetivo da pesquisa foi avaliar a relação entre os níveis de estresse, valência e previsibilidade de eventos de vida em relação ao índice de ansiedade e depressão de estudantes de uma universidade privada no nordeste da Colômbia. Trabalhamos com metodologia quantitativa, com delineamento transversal não experimental e escopo descritivo-correlacional. A amostra foi não probabilística por conveniência, composta por 33.7\% $(n=186)$ de homens e $66.3 \%(n=366)$ de mulheres, com média de idade de 18.9 anos. As variáveis do estudo foram avaliadas com: Inventário de Depressão de Beck II, Inventário de Ansiedade de Beck e Questionário de Eventos Vitais. Foram encontradas correlações estatisticamente significativas entre os níveis de ansiedade e depressão com valência negativa e a previsibilidade do evento. Esses resultados apoiam a teoria do desamparo-desesperança e discordam das evidências científicas existentes sobre 0 assunto.
\end{abstract}

Palavras chave

Stress, estudantes universitários, eventos de vida, ansiedade, depressão. 


\section{ntroducción}

El estrés es parte de la vida cotidiana de la humanidad desde antes del surgimiento de la civilización (Gálvez, 2005). Uno de los grandes retos de la ciencia contemporánea es reducir los niveles de estrés en la población a nivel mundial y la afectación que estos generan sobre la salud (Moscoso, 2009). Dependiendo de sus síntomas, cronicidad, origen o malestar, el estrés puede tener diferentes clasificaciones como: estrés hospitalario o prequirúrgico, postraumático, distrés, eustrés, académico, burnout, crónico y agudo (Corzo, 2009; Madrigal et al., 2005; Marrau, 2004; Naranjo, 2009; Rodríguez-Fernández, García-Acero \& Franco, 2013; Suárez-Montes \& DíazSubieta, 2015).

Chiriboga (1989) clasificó el estrés en tres niveles: el primer nivel llamado "micro" lo constituyen aquellos eventos que se dan en la vida diaria de las personas como: embotellamientos, levantarse tarde para ir al trabajo, no completar el examen a tiempo, entre otros; el segundo nivel, denominado "mazo", abarca un conjunto de situaciones que marcan mayores picos de estrés, como: una enfermedad, la muerte de un amigo y/o problemas laborales o de pareja; por último, el tercer nivel conocido como "macro", considera aquellas situaciones que afectan a los habitantes de una comunidad o nación, como, por ejemplo, crisis económicas o humanitarias. De acuerdo con Lazarus \& Folkman (1984) cuando una persona se enfrenta a una de las anteriores situaciones, se genera un malestar psicológico al percibir que las exigencias del entorno exceden sus capacidades y recursos, a partir de lo cual inicia una evaluación que determina el grado de estrés que está experimentando. Naranjo (2009), por su parte, sostiene que el estrés surge a partir de la evaluación cognitiva que la persona hace del evento considerando aspectos internos y los propios del ambiente. Así mismo, se ha demostrado que algunas situaciones estresantes pueden dar origen a diferentes enfermedades de carácter agudo o crónico debido al impacto de estos eventos, que dependen de la edad, el género, la red de apoyo y la valoración final que se le otorgue a lo vivido: estas situaciones son conocidas como sucesos vitales estresantes (Muratori, Delfino, \& Zubieta, 2012). Por su parte, Suárez (2010) los entiende como experiencias importantes en la vida de la persona que promueven cambios evolutivos. No obstante, pueden ser vistos como situaciones demandantes que sobrepasan los recursos de quien las enfrenta (Guzmán \& Álvarez, 2009).

Por lo expuesto previamente, resulta decisiva la valoración que la persona hace del suceso que vivió y las consecuencias sobre su salud mental dependerán de la connotación que le otorgue (Holmes \& Rahe, 1967, citados en González \& Morera, 1983). De igual forma, Barcelata, Durán y Gómez-Maqueo (2012) explican que "el desequilibrio entre la percepción y la evaluación de un suceso, y de los recursos disponibles para enfrentarlo, es lo que da pauta para que una experiencia sea interpretada como negativa o estresante" (p. 514). Por tal razón, la manera como se percibe un suceso puede resultar más significativa que el mismo hecho, lo que conlleva a que estas situaciones puedan ser generadoras 
de sintomatología depresiva y/o ansiosa (Veytia, González, Andrade, \& Oudhof, 2012). En este mismo sentido, Guzmán y Álvarez (2009) afirman que cuando se le otorga una evaluación de tipo negativo al impacto emocional, éste se puede asociar a sintomatología depresiva; los autores también sostienen que la depresión tiene mayor presencia en los sujetos que han sido partícipes de eventos calificados como negativos y estresantes.

Al respecto, la depresión es entendida como el trastorno psicológico de mayor prevalencia en Colombia (Ministerio de Salud, 2017). Dicho término hace referencia a un conjunto de síntomas caracterizado por un estado de abatimiento, sentimiento de tristeza, impacto en el comportamiento, niveles de actividad y de pensamiento, incluso puede llevar al acto suicida (Diulio et al., 2015; World Health Organization, 2014). A partir de lo anterior, se tiene en cuenta la sintomatología, la intensidad, cronicidad y curso para determinar la existencia o no del trastorno (Caballo, 2006; Londoño \& González, 2016). Por otra parte, el término ansiedad hace referencia a la expresión psicofisiológica persistente y difusa, con probabilidad de alcanzar el pánico ante una situación que se experimenta y anticipa como amenazante (Piqueras et al., 2008; Sierra, Ortega, \& Zubeidat, 2003).

Infortunadamente, en los últimos años se ha presentado un crecimiento en la prevalencia de enfermedades mentales entre los adultos jóvenes a nivel mundial (González \& Rego, 2006; Ministerio de Salud, 2017; Organización Mundial de la Salud, 2013). Se ha evidenciado que la ansiedad y la depresión afectan el desarrollo normal teniendo un impacto negativo en el rendimiento académico y en el desempeño psicosocial (Meyer, Ramírez, \& Pérez, 2013; Román, Vinet, \& Alarcón, 2014). Así mismo, la universidad constituye una etapa de la vida donde los estudiantes son adolescentes o adultos jóvenes, que buscan concretar sus proyectos de vida, adquiriendo la mayoría de edad y asumiendo nuevas responsabilidades sociales (CardonaArias, Pérez-Restrepo, Rivera-Ocampo, \& Gómez-Martínez, 2015). Por tanto, la universidad ha resultado ser un entorno que representa un conjunto de situaciones estresantes para el individuo, ya que el estudiante puede enfrentarse a una falta de control sobre el nuevo ambiente que resulta ser generador de estrés, desencadenando en el fracaso académico universitario (Noriega et al., 2014). Por tal razón, el objetivo de esta investigación fue evaluar la relación entre los niveles de estrés, la valencia y la predictibilidad de sucesos vitales en relación con el índice de ansiedad y depresión de estudiantes de una universidad privada en el nororiente de Colombia.

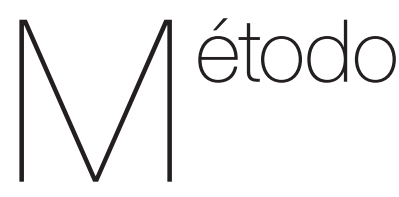

La presente investigación se realizó bajo un enfoque metodológico cuantitativo de alcance descriptivo-correlacional, con un diseño no experimental de corte transversal. La selección de la muestra se llevó a cabo a partir de un muestreo no probabilístico a conveniencia. La muestra estuvo constituida por 552 estudiantes, conformada por un 33.7\% ( $\mathrm{n}=$ 
186) hombres y el $66.3 \%(n=366)$ mujeres, de los cuales el 28.4\% ( $n=157)$ cursaba primer semestre; el 33.3\% $(n=184)$ se hallaban en tercer semestre (33.3\%), el $17.8 \%(n=98)$ se encontraba en quinto semestre; por último, el 20.5\% ( $n=113$ ) cursaba séptimo semestre. Con respecto al estado civil, el $97.1 \%(n=536)$ de los participantes estaban solteros, el 2.2\% ( $n=12)$ se encontraba en unión libre y el $.7 \%(n=4)$ estaban casados. Sus edades oscilaron entre los 17 a 33 años, con una media de 18.95 ( $\mathrm{DE}=2.09$ ).

\section{Instrumentos}

Para cumplir con el objetivo propuesto en esta investigación, se utilizaron tres instrumentos:

Inventario de Ansiedad de Beck versión español

(BAl; Estrada, Delgado, Landero, \& González, 2013), consta de 21 ítems con cuatro opciones de respuesta $(1=$ Nada, 2 = Levemente, 3 = Moderadamente y 4 = Severamente) que mide los síntomas de la ansiedad, referidos a la semana previa y al momento actual de la aplicación; así mismo, posee un alfa de Cronbach .90.

\section{Inventario de Depresión de Beck versión española}

(BDI-II; Sanz, 2013; Sanz, Navarro \& Vázquez, 2003; Sanz, Perdigón, \& Vázquez, 2003) es una escala que consta de 22 ítems de opción múltiple con única respuesta que permite evaluar los síntomas depresivos referidos a la semana previa y al momento actual de la aplicación; tiene un alfa Cronbach de .89.

\section{Cuestionario de Sucesos Vitales}

(CSV; Sandín \& Charot, 2017), con un alfa de .082, consiste en una lista de 60 sucesos, permitiendo la evaluación del estrés psicosocial y la frecuencia de sucesos vitales, experimentados durante el último año. En este último instrumento, el sujeto debe contestar indicado el grado de estrés que va en una escala de 1 (nada o muy poco), 2 (bastante), 3 (mucho) y 4 (muchísimo) y reportando si el suceso fue positivo, negativo, esperado o inesperado.

\section{Procedimiento}

En primera instancia, se informó mediante una carta el objetivo y las características del estudio a los directivos de 10 programas académicos de una universidad privada de Bucaramanga, Colombia. En segundo lugar, se les explicó a los estudiantes voluntarios los aspectos relacionados con la confidencialidad, que incluye el consentimiento informado y asentimiento, considerando las disposiciones éticas exigidas para la investigación con seres humanos, según la ley 1090 (2006) que rige el actuar ético del psicólogo en Colombia. Posteriormente, durante el primer y segundo semestre del año 2017, los participantes contestaron vía web la batería de instrumentos que se construyó en la plataforma LimeSurvey.

\section{Análisis de Datos}

Para el análisis de datos, se utilizó el software Statistical Package for the Social Sciences (SPSS) versión 25. Se aplicó un análisis univariable para la caracterización de la muestra. Después, se ejecutó la 
prueba Kolmogorov-Smirnov para evaluar la distribución de normalidad del conjunto de datos cuantitativos. Teniendo en cuenta la distribución no normal en los datos cuantitativos, se emplearon estadísticos no paramétricos.

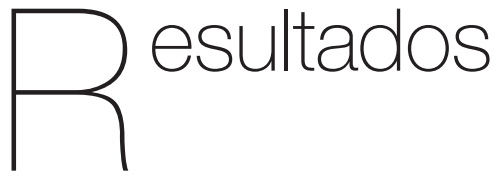

Con relación a los sucesos vitales más frecuentes, el $76.2 \%(n=421)$ de los participantes experimentaron problemas legales graves; un $72.4 \%(n=400)$ vivenciaron una lesión física importante y el 68.4\% ( $\mathrm{n}$ $=378$ ) indicaron problemas con sus superiores en el trabajo. En contraparte, los tres sucesos vitales menos frecuentes por los estudiantes fueron: tener relaciones sexuales fuera del matrimonio por el $22.6 \%$ ( $n=125)$; un $22.4 \%(n=124)$ experimentaron la enfermedad de un miembro de la familia y el $21.5 \%$ ( $n=119)$ vivenciaron la muerte de un amigo. Por otro lado, los tres sucesos vitales más categorizados con el grado "muchísimo estrés" fueron: el 15.2\% ( $n=84)$ a exámenes importantes; un $14.3 \%(n=79)$ a la muerte de algún familiar cercano y el $12.8 \%(n=$ 71) a problemas académicos. Finalmente, se identificó que los tres sucesos vitales con mayor clasificación como negativos fueron: el 48.9\% ( $n=270)$ a problemas con superiores del trabajo; un 45.4\% (n $=251$ ) al repentino y serio deterioro de la audición o visión y el 44.9\% $(n=248)$ a enamorarse o iniciar una amistad íntima y profunda. En la Tabla 1 se encuentran los datos descriptivos sobre las medidas de tendencia central de las variables cuantitativas.

Tabla 1.

Medidas de tendencia central de las variables cuantitativas

\begin{tabular}{cccccc}
\hline & Media & Mediana & DE & Mínimo & Máximo \\
\hline Edad & 18.95 & 19.00 & 2.09 & 16 & 33 \\
BAI & 11.62 & 9.00 & 9.85 & 0 & 55 \\
BDI-II & 8.96 & 7.00 & 8.36 & 0 & 43 \\
SCV & 44.64 & 35.00 & 36.79 & 0 & 169 \\
\hline
\end{tabular}

Nota: BAI: Inventario de Ansiedad de Beck versión español; CSV: Cuestionario de Sucesos Vitales; BDI-II: Inventario de Depresión de Beck versión española Fuente: Elaboración propia.

Respecto al Rho de Spearman, se evidenció una relación estadísticamente significativa entre los niveles de depresión y ansiedad $(r=.70, p=.00)$; niveles de estrés y ansiedad ( $r=.13, p=.00$ ). Sin embargo, no se identificó una relación estadísticamente significativa entre los niveles de estrés y depresión $(r=.06, p=.11)$. Por otra parte, en la Tabla 2 se presentan los datos de correlación significativas entre los niveles de estrés de los sucesos vitales, los niveles de ansiedad y depresión. 
Tabla 2.

Rho de Spearman significativas entre los niveles de estrés de los sucesos vitales y los niveles de ansiedad y depresión.

\begin{tabular}{|c|c|c|}
\hline Suceso vital & BDA & BDI-II \\
\hline Problemas con colegas o compañeros de trabajo & $.25^{*}$ & $.20^{*}$ \\
\hline Aumento en las responsabilidades económica & $-.14^{*}$ & $-.16^{*}$ \\
\hline Problemas legales graves & $.22^{*}$ & $.18^{*}$ \\
\hline Problemas legales relacionados con el alcohol o las drogas & $-.12^{*}$ & $-.22^{*}$ \\
\hline Cambio de lugar de trabajo & $.17^{*}$ & $.13^{*}$ \\
\hline Muerte de un amigo íntimo & $-.13^{*}$ & $.21^{*}$ \\
\hline Relaciones sexuales fuera del matrimonio & $-.12^{*}$ & $-.21^{*}$ \\
\hline Cambio de casa & $-.13^{*}$ & $-.15^{*}$ \\
\hline Muerte de algún familiar cercano & $-.15^{\star}$ & $-.25^{*}$ \\
\hline Préstamo o hipoteca de más de 100 mil euros & $.20^{*}$ & $.21^{*}$ \\
\hline Repentino y serio deterioro de la audición o visión & $.29^{*}$ & $.25^{*}$ \\
\hline Lesión física importante & $.27^{\star}$ & $.22^{*}$ \\
\hline Enamorarse 0 iniciar una amistad íntima y profunda & $.13^{*}$ & .15 \\
\hline Enfermedad de un miembro de la familia & $-.16^{*}$ & $-.25^{*}$ \\
\hline Problemas con superiores en el trabajo & $.29^{*}$ & $.31^{*}$ \\
\hline Éxito personal importante & $-.13^{*}$ & $-.15^{\star}$ \\
\hline
\end{tabular}

Nota: *: Correlación estadísticamente significativa al nivel del 95\%. BAl: Inventario de Ansiedad de Beck versión español; BDI-II: Inventario de Depresión de Beck versión española; Fuente: Elaboración propia.

Por otra parte, en la Tabla 3 se mues- niveles de ansiedad y depresión según la tran los resultados, estadísticamente valencia de los eventos experimentados significativos, en la comparación de los por los participantes.

Tabla 3.

Comparación de los niveles de depresión y ansiedad según la valencia otorgada a los eventos experimentados.

\begin{tabular}{|c|c|c|c|c|}
\hline Variable & Evento & Positivo n (RP) & Negativo $\mathrm{n}(\mathrm{RP})$ & U (valor-p) \\
\hline \multirow{7}{*}{ 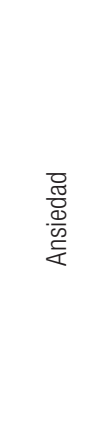 } & Ascenso en el trabajo & $33(116.08)$ & $156(90.54)$ & $1878.50(.01)$ \\
\hline & Comprar una casa & $85(56.44)$ & $46(83.66)$ & $1142.50(.00)$ \\
\hline & Ruptura de noviazgo o relación similar & $21(121.50)$ & $163(88.76)$ & $1102.50(.00)$ \\
\hline & Problema legal en una pelea & $187(116.46)$ & $55(138.64)$ & $4200.00(.03)$ \\
\hline & Cambio de casa & $106(72.97)$ & $50(90.22)$ & $1960.50(.00)$ \\
\hline & Repentino y serio deterioro de la audición o visión & $53(176.81)$ & $251(147.37)$ & $5363.00(.02)$ \\
\hline & Lesión física importante & $256(172.29)$ & $102(197.58)$ & $11211.50(.03)$ \\
\hline
\end{tabular}


Andres Julian Usuga Jerez, Nancy Viviana Lemos Ramírez,

pp • 61-74 Jessica Lizeth Pinzón Ardila, Paula Fernanda Pérez Rivero y Ana Fernanda Uribe Rodríguez

Continuación

\begin{tabular}{|c|c|c|c|c|}
\hline Variable & Evento & Positivo n (RP) & Negativo $\mathrm{n}(\mathrm{RP})$ & U (valor-p) \\
\hline \multirow{5}{*}{ Ansiedad } & Reconciliación matrimonial o de pareja & $22(93.93)$ & $120(67.39)$ & $826.50(.00)$ \\
\hline & Separarse de los padres & $40(155.05)$ & $2214(122.35)$ & $3178.00(.01)$ \\
\hline & Fracaso en algún negocio importante & $21(108.14)$ & $143(78.73)$ & $963.00(.00)$ \\
\hline & Cambio de centro de estudios & $291(163.54)$ & $53(221.71)$ & $5103.50(.00)$ \\
\hline & Hospitalización de algún familiar & $30(96.10)$ & $129(76.26)$ & $1452.00(.03)$ \\
\hline \multirow{22}{*}{ 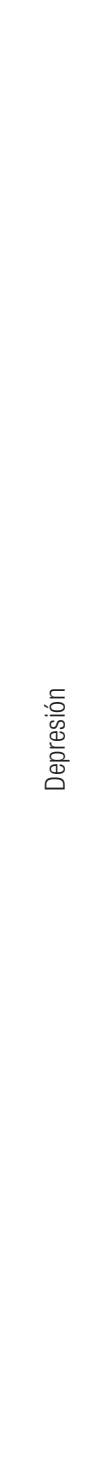 } & Aumento en las responsabilidades económicas & $29(77.48)$ & $93(56.52)$ & $885.00(.00)$ \\
\hline & Exámenes importantes & $45(141.18)$ & $202(120.17)$ & $3400.50(.00)$ \\
\hline & Reducción sustancial en los ingresos & $134(108.17)$ & $92(121.27)$ & $5044.50(.02)$ \\
\hline & Ascenso en el trabajo & $33(123.88)$ & $156(88.89)$ & $1621.00(.00)$ \\
\hline & Un miembro de la familia deja la casa familiar & $145(88.71)$ & $40(108.54)$ & $2278.50(.03)$ \\
\hline & Comprar una casa & $85(57.50)$ & $46(81.71)$ & $1232.50(.00)$ \\
\hline & Ruptura de noviazgo o relación similar & $21(125.50)$ & $163(88.22)$ & $1014.00(.00)$ \\
\hline & Muerte de un amigo íntimo & $12(69.38)$ & $85(46.12)$ & $265.50(.00)$ \\
\hline & Muerte de un hijo & $41(88.16)$ & $94(59.21)$ & $1100.50(.00)$ \\
\hline & Problema legal en una pelea & $187(116.17)$ & $55(139.64)$ & $4145.00(.02)$ \\
\hline & Cambio de casa & $106(72.97)$ & $50(90.22)$ & $2064.00(.00)$ \\
\hline & Enfermedad o accidente que requiera guardar cama & $30(110.90)$ & $162(93.83)$ & $1639.50(.00)$ \\
\hline & Accidente de tráfico & $31(103.98)$ & $171(101.05)$ & $1982.00(.02)$ \\
\hline & Repentino y serio deterioro de la audición o visión & $53(178.71)$ & $251(146.97)$ & $5262.50(.02)$ \\
\hline & Lesión física importante & $256(168.65)$ & $102(206.73)$ & $10279.00(.00)$ \\
\hline & $\begin{array}{l}\text { Cambio de horario o de las } \\
\text { condiciones del trabajo actual }\end{array}$ & $82(64.66)$ & $57(77.68)$ & $1705.50(.00)$ \\
\hline & Vivir temporalmente fuera del hogar & $61(101.44)$ & $112(79.13)$ & $2535.00(.00)$ \\
\hline & Reconciliación matrimonial o de pareja & $22(897.55)$ & $120(66.73)$ & $747.00(.00)$ \\
\hline & Separarse de los padres & $40(149.85)$ & $214(123.32)$ & $3386.00(.03)$ \\
\hline & Fracaso en algún negocio importante & $21(110.21)$ & $143(78.43)$ & $919.50(.00)$ \\
\hline & $\begin{array}{l}\text { Pérdidas económicas importantes } \\
\text { no asociadas al trabajo }\end{array}$ & $27(86.39)$ & $97(55.85)$ & $664.50(.00)$ \\
\hline & Cambio de centro de estudios & $291(165.13)$ & $53(221.97)$ & $5566.50(.00)$ \\
\hline
\end{tabular}

Nota: RP = Rango Promedio; U = Prueba U de Mann-Whitney; Fuente: Elaboración propia.

Finalmente, en la Tabla 4 se de- niveles de ansiedad y depresión según la muestran las diferencias, estadística- predictibilidad.

mente significativas, halladas entre los 
Tabla 4.

Comparación de los niveles de ansiedad y depresión según la predictibilidad otorgada a los eventos experimentados.

\begin{tabular}{|c|c|c|c|c|}
\hline Variable & Evento & Esperado n (RP) & Inesperado n (RP) & U (valor-p) \\
\hline \multirow{15}{*}{$\begin{array}{l}\frac{0}{0} \\
\frac{\pi}{0} \\
\frac{1}{10} \\
\frac{1}{4}\end{array}$} & Exámenes importantes & $69(123.34)$ & $151(104.63)$ & $4323.50(.04)$ \\
\hline & Reducción sustancial en los ingresos & $124(99.35)$ & $93(121.87)$ & $4569.00(.00)$ \\
\hline & $\begin{array}{l}\text { Problemas legales relacionados } \\
\text { con el alcohol o las drogas }\end{array}$ & $52(49.14)$ & $75(74.30)$ & $1177.50(.00)$ \\
\hline & Ascenso en el trabajo & $25(107.44)$ & $146(82.33)$ & $1289.00(.01)$ \\
\hline & Un miembro de la familia deja la casa familiar & $98(68.24)$ & $58(95.83)$ & $1837.00(.00)$ \\
\hline & Cambio de Casa & $69(62.60)$ & $82(87.27)$ & $1904.50(.00)$ \\
\hline & $\begin{array}{l}\text { Problemas con vecinos o con familiares } \\
\text { que no viven en la casa }\end{array}$ & $51(121.12)$ & $159(100.49)$ & $3258.00(.03)$ \\
\hline & Nuevo empleo laboral & $199(136.39)$ & $90(164.04)$ & $7241.00(.00)$ \\
\hline & Finalización de estudios & $47(141.89)$ & $175(103.34)$ & $2684.00(.00)$ \\
\hline & Enamorarse o iniciar una amistad íntima y profunda & $54(150.32)$ & $206(125.30)$ & $4491.50(.02)$ \\
\hline & Ruptura de relaciones sexuales fuera del matrimonio & $29(75.47)$ & $90(55.02)$ & $856.50(.00)$ \\
\hline & $\begin{array}{l}\text { Cambio de horario o de las } \\
\text { condiciones del trabajo actual }\end{array}$ & $56(66.50)$ & $62(53.18)$ & $1344.00(.03)$ \\
\hline & Reconciliación matrimonial o de pareja & $36(83.33)$ & $103(65.34)$ & $1374.00(.02)$ \\
\hline & Separarse de los padres & $46(146.37)$ & $185(108.45)$ & $2858.00(.00)$ \\
\hline & Cambio de centro de estudios & $241(155.57)$ & $82(180.91)$ & $8330.50(.03)$ \\
\hline \multirow{13}{*}{ 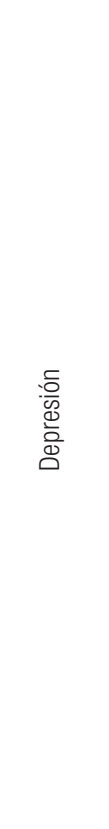 } & Aumento en las responsabilidades económica & $36(65.25)$ & $68(45.75)$ & $765.00(.00)$ \\
\hline & Reducción sustancial en los ingresos & $124(101.01)$ & $93(119.65)$ & $4775.50(.03)$ \\
\hline & $\begin{array}{l}\text { Problemas legales relacionados } \\
\text { con el alcohol o las drogas }\end{array}$ & $52(54.54)$ & $75(70.56)$ & $1458.00(.01)$ \\
\hline & Ascenso en el trabajo & $25(111.50)$ & $146(81.63)$ & $1187.50(.00)$ \\
\hline & Un miembro de la familia deja la casa familiar & $98(71.94)$ & $58(89.59)$ & $2199.00(.01)$ \\
\hline & Muerte de un amigo íntimo & $25(59.14)$ & $73(46.20)$ & $671.500(.04)$ \\
\hline & Cambio de Casa & $69(61.50)$ & $82(88.20)$ & $1828.50(.00)$ \\
\hline & Muerte de algún familiar cercano & $34(47.50)$ & $82(63.06)$ & $1020.00(.02)$ \\
\hline & Periodo de fiestas & $28(87.30)$ & $221(129.78)$ & $2038.50(.00)$ \\
\hline & Sufrir un robo & $20(56.58)$ & $157(93.13)$ & $921.50(.00)$ \\
\hline & Finalización de estudios & $47(139.16)$ & $175(104.07)$ & $2812.50(.00)$ \\
\hline & Separarse de los padres & $46(147.15)$ & $185(108.25)$ & $2822.00(.00)$ \\
\hline & Cambio de centro de estudios & $241(155.05)$ & $82(182.43)$ & $8205.50(.02)$ \\
\hline
\end{tabular}

Nota: RP = Rango Promedio; U = Prueba U de Mann-Whitney; Fuente: Elaboración propia. 


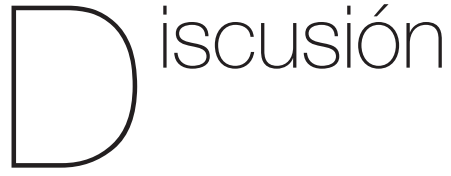

Tomando en cuenta la relación entre los niveles de estrés y los índices de ansiedad, se identificó una correlación débil, pero estadísticamente significativa, lo cual diverge de la mayoría de los estudios sobre este tema, que evidencian una relación fuerte (Tijerina et al., 2018; CaballeroDomínguez \& Suárez-Colorado, 2019). De otra parte, no se evidenció una relación estadísticamente significativa entre los niveles de estrés y los índices de depresión, lo que discrepa de la literatura científica (Caballero-Domínguez \& Suarez-Colorado, 2019; Emiro et al., 2018; Guerrero et al., 2013; Gutiérrez et al., 2010; Tijerina et al., 2018). De tal forma, se concluye que los participantes del estudio presentan mayor sintomatología ansiosa que depresiva, esto coincide con Arrieta, Díaz y González (2014) y Dávila, Ruiz, Moncada y Gallardo (2011). Dicho hallazgo puede deberse a que los universitarios refieren síntomas ansiosos transitorios como respuesta a las exigencias que requieren para el programa académico y la vida diaria. Por último, se identificó una relación estadísticamente significativa entre los niveles de ansiedad y depresión, lo cual reitera la comorbilidad de estas variables documentadas en la literatura científica (Arrieta et al., 2014; Balanza, Morales, \& Guerrero, 2009; Caballero-Domínguez \& Suárez-Colorado, 2019; Gorman, 1996; Ninan \& Berger, 2001).

Por otra parte, la teoría reformulada de la indefensión (Abramson et al., 2002) y la teoría de indefensión-desesperanza de la ansiedad y de la depresión (Alloy, Kelly,
Mineka, \& Clements, 1990), dan a conocer que los eventos estables con pocas probabilidades de que cambien y categorizados como negativos pueden inducir a sentimientos de desesperanza y paralelamente, a síntomas de depresión. De lo anterior, los resultados del presente trabajo apoyan dichos planteamientos, puesto que ciertos sucesos vitales categorizados como negativos se encontraron relacionados con mayores niveles de sintomatología depresiva experimentada por los participantes, al igual que se evidencia en otras investigaciones (Camuñas, Mavrou, \& Miguel-Tobal, 2019; Gómez et al., 2018; Sanjuán, Magallares, González, \& PérezGarcía, 2013). Por último, el modelo de Alloy et al. (1990) explica que la ansiedad pura inicia de un sentimiento incierto de indefensión debido a consecuencias futuras; cuando esa expectativa incierta se hace cierta, surge un síndrome mixto de ansiedad y depresión que, posteriormente, puede llevar a la depresión pura. Ahora bien, los hallazgos de la predictibilidad "inesperada" de ciertos sucesos vitales estresantes están relacionados con mayores niveles de ansiedad, encontrando similitud con diferentes investigaciones donde identifican esta asociación con cuadros de ansiedad (Barlow, 2008; Thomson, Hood, \& Short, 2016).

Debido a limitaciones metodológicas y estadísticas no es posible establecer una lógica ni un orden causal entre estas variables. Por otra parte, se encuentra un campo de investigación abierto con relación al estrés percibido, las estrategias de afrontamiento hacia el estrés y las estrategias de regulación emocional que llevan a cabo los estudiantes al momento de haber experimentado ciertos sucesos vitales. A pesar de estas restricciones, el presente trabajo ha contribuido a conocer 
de forma detallada la asociación que existe entre la valencia y la predictibilidad de sucesos vitales estresantes con relación a los índices de ansiedad y depresión en estudiantes universitarios. Así mismo, esta investigación contribuye a completar y profundizar en este interesante campo de estudio del bienestar emocional, teniendo repercusiones importantes tanto teóricas como aplicadas con la población universitaria.

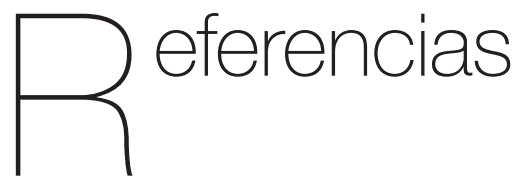

Abramson, L., Alloy, L., Hankin, B., Haeffel, G., MacCoon, D., \& Gibb, B. (2002). Cognitive vulnerability-stress models of depression in a self-regulatory and psychobiological context. New York, USA: The Guilford Press.

Alloy, L., Kelly, K., Mineka, S., \& Clements, C. (1990). Comorbidity in anxiety and depressive disorders: A helplessness/ hopelessness perspective. In J. D. Maser \& C. R. Cloninger (Eds.), Comorbidity of mood and anxiety disorders (pp. 499-543). Washington, DC: American Psychiatry Press

Arrieta, K., Díaz, S. \& González, F. (2014). Síntomas de depresión y ansiedad en jóvenes universitarios: prevalencia y factores relacionados. Revista Clínica de Medicina de Familia, 7(1), 14-2.

Balanza, S., Morales, I. \& Guerrero, J. (2009). Prevalencia de Ansiedad y Depresión en una Población de Estudiantes
Universitarios: Factores Académicos y Sociofamiliares Asociados. Clínica y Salud, 20(2), 177-187.

Barcelata, B., Durán, C., \& Gómez-Maqueo, E. (2012). Valoración subjetiva de los sucesos de vida estresantes en dos grupos de adolescentes de zonas marginadas. Salud Mental, 35(6), 513-520.

Barlow, D. (2008). Anxiety adn its disorders: the nature and treatment of anxiety and panic. New York: The Guilford Press.

Caballero-Domínguez, C., \& Suárez-Colorado, Y. (2019). Tipologías distintivas de respuestas ante el estrés académico en universitarios: un análisis de clasificación. Duazary, 16(2) 245-257. https://doi. org/10.21676/2389783X.2748

Caballo, V. (2006). Manual para la evaluación clínica de los trastornos psicológicos. Trastornos de la edad adulta e informes psicológicos. Madrid, España: Pirámide.

Camuñas, N., Mavrou, I. \& Miguel-Tobal, J. (2019). Ansiedad y tristeza-depresión: Una aproximación desde la teoría de la indefensión-desesperanza. Revista de Psicopatología y Psicología Clínica, 24, 19-28.

Cardona-Arias, J., Pérez-Restrepo, D., RiveraOcampo, S., Gómez-Martínez, J \& Reyes, J. (2015). Prevalencia de ansiedad en estudiantes universitarios. Diversitas: Perspectivas en Psicología, 11(1), 79-89.

Chiriboga, D. A. (1989). The measurement of stress exposure in later life. En K. S. Markides \& C. L. Cooper (Eds.), Aging, stress and health (pp. 13-41). Chichester, Inglaterra: John Wiley \& Sons 
Congreso de Colombia. (6 de septiembre de 2006). De la profesión de psicología. [Ley 1090 de 2066]. Recuperado de ttp://www. sociedadescientificas.com/userfiles/file/ LEYES/1090\%2006.pdf

Corzo, P. (2009). Trastorno por estrés postraumático en psiquiatría militar. Revista de la Facultad de Medicina, 17(1), 81-86.

Dávila, A., Ruiz, R., Moncada, L., \& Gallardo, I. (2011). Niveles de ansiedad, depresión y percepción de apoyo social en estudiantes de Odontología de la Universidad de Chile. Revista de Psicología, 20(2), 147-172.

Diulio, A., Dutta, N., Gauthier, J., Witte, T., Correia, C., \& Angarona, D. (2015). Associations among depressive symptoms, drinking motives, and risk for alcohol-related problems in veterinary students. The Journal of Veterinary Medical Education, 42, 11-17.

Emiro, J., Amador, O., Calderón, V., Castañeda, T., Osorio, Y., \& Díez, P. (2018). Depresión y su relación con el consumo de sustancias psicoactivas, el estrés académico y la ideación suicida en estudiantes universitarios colombianos. Revista Health and Addictions/Salud y Drogas, 18(2), 227-239

Estrada, B., Delgado, C., Landero, R., \& González, M. (2013). Propiedades psicométricas del modelo factorial del BDI-II (versión española) en muestras mexicanas de población general y estudiantes universitarios. Universitas Psychologica, 14(1), 125-136.

Gálvez, J. (2005). Trastornos por estrés y sus repercusiones neuropsicoendocrinológicas. Revista Colombiana de Psiquiatría, 34(1), 77-100.
Gómez, Y., Ángel, J., Cañizares, C., Latting, M., Agudelo, D., Arenas, A., \& Ferro, E. (2018). El papel de la valoración de los sucesos vitales estresantes en el Trastorno Depresivo Mayor. En A. Melús (Presidencia) VIII Congreso Nacional de Psicología. Congreso llevado a cabo en Santiago, Chile.

González, B., \& Rego, E. (2006). Problemas emergentes en la salud mental de la juventud. Madrid, España: Instituto de Juventud.

González, J., \& Morera, F. (1983). La valoración de sucesos vitales: Adaptación española de la escala de Holmes y Rahe. Psiquis, 4(1) $7-11$

Gorman, J. (1996). Comorbid depression and anxiety spectrum disorders. Depression and Anxiety, 4, 160-168.

Guerrero, J., Heinze, G., Ortiz de León, S., Cortés, J., Barragán, V., \& Flores-Ramos, M. (2013). Factores que predicen depresión en estudiantes de medicina. Gaceta Médica de México, 149, 598-604.

Gutiérrez, J., Montoya, L., Toro, B., Briñón, M., Rosas, E. \& Salazar, L. (2010). Depresión en estudiantes universitarios y su asociación con el estrés académico. CES Medicina, 24(1), 7-17.

Guzmán, C., \& Álvarez, R. (2009). Sucesos vitales y factores de riesgo asociados: el caso de pacientes con cáncer. Revista Psicología y Salud, 1(19), 21-32.

Lazarus, R., \& Folkman, S. (1984). Stress, appraisal and coping. New York: Springer.

Londoño, C., \& González, M. (2016). Relevancia de la depresión y factores asociados en 
hombres. Acta Colombiana de Psicología, 19(2), 315-329.

Madrigal, I., Moreno, J., Rubio A., Ibañez A., López, J., \& Martínez. L., (2005). Respuesta al estrés prequirúrgico en la cirugía sin ingreso: efectos sobre las poblaciones linfocitarias de un procedimiento de psicoprofilaxis quirúrgica. Revista Española de Anestesiología y Reanimación, 52(7), 383-388.

Marrau, C. (2004). El síndrome de Burnout y sus posibles consecuencias en el trabajador docente. Fundamentos en Humanidades, 5(1), 53-68.

Meyer K., A., Ramírez F., L., \& Pérez V., C. (2013). Percepción de estrés en estudiantes chilenos de Medicina y Enfermería. Revista de Educación en Ciencias de la Salud, 10(2), 79-85.

Ministerio de salud. (2017). Boletín de salud mental Depresión Subdirección de Enfermedades No Transmisibles. Recuperado de https://www.minsalud.gov. co/sites/rid/Lists/BibliotecaDigital/RIDE/ VS/PP/ENT/boletin-depresion-marzo-2017. pdf

Moscoso, M. (2009). De la mente a la célula, impacto del estrés en la psiconeuroendocrinología. Liberabit. Revista de Psicología, 15(2), 143-152.

Muratori, M., Delfino, G., \& Zubieta, E. (2012). Sucesos Vitales y bienestar. Anuario de Investigaciones, 19, 49-57.

Naranjo, M. (2009). Una revisión teórica sobre el estrés y algunos aspectos relevantes de éste en el ámbito educativo. Revista Educación, 33(2), 171-190.
Ninan, P., \& Berger, J. (2001). Symptomatic and syndromal anxiety and depression. Depression and Anxiety, 14, 79-85.

Noriega, J., Castellanos, J., Orejuela, D., Cepeda, D., Barajas-Soto, S., \& UribeRodríguez, A. (2014). Sucesos vitales en jóvenes universitarios de la Universidad Pontificia. Apuntes de Investigación, 9, 1-16.

Organización Mundial de la Salud (2013). Plan de acción sobre salud mental 2013-2020. Recuperado de https://apps.who.int/iris/bitstream/ handle/10665/97488/9789243506029_ spa.pdf;jsessionid=BB79E17A8B744EEFA 3C6E26D8690262D?sequence=1

Piqueras, J., Martínez, A., Ramos, V., Rivero, R., García, L., \& Oblitas, A. (2008). Ansiedad, depresión y salud. Suma Psicológica, 15(1), 43-73.

Rodríguez-Fernández, J., García-Acero, M., \& Franco, P. (2013). Neurobiología del estrés agudo y crónico: su efecto en el eje hipotálamo-hipófisis-adrenal y la memoria. Universitas Médica, 54(4), 472-494.

Román, F., \& Vinet, E., \& Alarcón, A. (2014). Escalas de Depresión, Ansiedad y Estrés (DASS-21): Adaptación y propiedades psicométricas en estudiantes secundarios de temuco. Revista Argentina de Clínica Psicológica, 23(2), 179-190

Sandín, B., \& Charot, P. (2017). Cuestionario de Sucesos Vitales (CSV): Estructura factorial, propiedades psicométricas y datos normativos. Revista de Psicopatología y Psicología Clínica, 22(2), 95-115.

Sanjuán, P., Magallares, A., González, J., \& Pérez-García, A. (2013). Estudio de la validez 
de la versión española del cuestionario de estilo atribucional ante situaciones negativas. Revista de Psicopatología y Psicología Clínica, 18, 61-71.

Sanz, J. (2013). 50 años de los inventarios de depresión de Beck: consejos para la utilización de la adaptación española del BDI-II en la práctica clínica. Papeles del Psicólogo, 34(3), 161-168.

Sanz, J., Navarro, M., \& Vázquez, C. (2003). Adaptación española del inventario para la depresión de beck-II (BDI-II):1 Propiedades psicométricas en estudiantes universitarios. Análisis y Modificación de Conducta, 29(124), 239-288.

Sanz, J., Perdigón, L., \& Vázquez, C. (2003). Adaptación española del Inventario para la Depresión de Beck-II (BDI-II):2. Propiedades psicométricas en población general. Clínica y Salud, 14, 249-280.

Sierra, J., Ortega, V., \& Zubeidat, I. (2003). Ansiedad, angustia y estrés: tres conceptos a diferenciar. Revista Mal-estar E Subjetividade, 3(1), 10-59.

Suárez, M. (2010). La importancia de los acontecimientos vitales estresantes en la práctica clínica. Revista Médica La Paz, 16(2) 58-62.
Suárez-Montes, N., \& Díaz-Subieta, I. (2015). Estrés académico, deserción y estrategias de retención de estudiantes en la educación superior. Revista Salud Pública, 17(2), 300-313.

Thomson, L., Hood, C., \& Short, S. (2016). Unpredictability and symptoms of depression and anxiety. Journal of Social and Clinical Psychology, 35(5), 371-385.

Tijerina, L., González, E., Gómez, M., Cisneros, M., Rodríguez, K., \& Ramos, E. (2018) Depresión, ansiedad y estrés en estudiantes de nuevo ingreso a la educación superior. Revista de Salud Pública y Nutrición, 17(4), 41-47

Veytia, M., González, N., Andrade, P., \& Oudhof, H. (2012). Depresión en adolescentes: papel de los sucesos vitales estresantes. Salud Mental, 35, 37-43.

World Health Organization. (2014). Preventing Suicide: a global imperative. Washington, DC: World Health Organization. 